\title{
Big Bang, Blowup, and Modular Curves: Algebraic Geometry in Cosmology ${ }^{\star}$
}

\author{
Yuri I. MANIN ${ }^{\dagger}$ and Matilde MARCOLLI ${ }^{\ddagger}$ \\ $\dagger$ Max-Planck-Institut für Mathematik, Bonn, Germany \\ E-mail:manin@mpim-bonn.mpg.de \\ $\ddagger$ California Institute of Technology, Pasadena, USA \\ E-mail: matilde@caltech.edu
}

Received March 01, 2014, in final form June 27, 2014; Published online July 09, 2014

http://dx.doi.org/10.3842/SIGMA.2014.073

\begin{abstract}
We introduce some algebraic geometric models in cosmology related to the "boundaries" of space-time: Big Bang, Mixmaster Universe, Penrose's crossovers between aeons. We suggest to model the kinematics of Big Bang using the algebraic geometric (or analytic) blow up of a point $x$. This creates a boundary which consists of the projective space of tangent directions to $x$ and possibly of the light cone of $x$. We argue that time on the boundary undergoes the Wick rotation and becomes purely imaginary. The Mixmaster (Bianchi IX) model of the early history of the universe is neatly explained in this picture by postulating that the reverse Wick rotation follows a hyperbolic geodesic connecting imaginary time axis to the real one. Penrose's idea to see the Big Bang as a sign of crossover from "the end of previous aeon" of the expanding and cooling Universe to the "beginning of the next aeon" is interpreted as an identification of a natural boundary of Minkowski space at infinity with the Big Bang boundary.
\end{abstract}

Key words: Big Bang cosmology; algebro-geometric blow-ups; cyclic cosmology; Mixmaster cosmologies; modular curves

2010 Mathematics Subject Classification: 85A40; 14N05; 14G35

\section{Introduction}

\subsection{Future and past boundaries of space-times}

The current observable domain of our expanding Universe is almost flat. Hence we assume that its good model is Minkowski's space-time. Therefore, its natural future boundary is modelled by (a domain in) the infinite three-dimensional hyperplane compactifying Minkowski 4-space to the projective 4 -space.

The Big Bang model of the beginning of our Universe postulates the special role of a certain "time zero" point in it, and we will argue that a natural past boundary is related to the algebraic geometric blow-up of this point.

We start with explaining the relevant geometry in more detail. Below $\mathbb{R}$ (resp. $\mathbb{C}$ ) always denotes the field of real (reps. complex) numbers. It is essential to keep track of the complex/algebraic geometry of our constructions and identify classical space-times as real points corresponding to certain real structures, as in Penrose's twistor program, description of instantons etc. Our main new contribution in this respect will be a description of cosmological time in the early Universe in terms of the geodesic flow on a modular curve.

Let $M^{4}$ be a 4-dimensional real linear space endowed with a non-degenerate symmetric quadratic form $q: S^{2}\left(M^{4}\right) \rightarrow \mathbb{R}$ of signature $(1,3)$ (that is, +--- ). The associated affine

\footnotetext{
*This paper is a contribution to the Special Issue on Noncommutative Geometry and Quantum Groups in honor of Marc A. Rieffel. The full collection is available at http://www.emis.de/journals/SIGMA/Rieffel.html
} 
space $\mathcal{M}^{4}$ (essentially, $M^{4}$ without marked zero point) with metric induced by $q$ is the standard model of Minkowski space-time (in which a time-orientation is not yet chosen explicitly). By construction, $M^{4}$ acts on $\mathcal{M}^{4}$ by shifts (and therefore it acts also on various subsets, e.g., affine subspaces of $\mathcal{M}^{4}$ ). If one subset is obtained by shift from another subset, we say that they are parallel. Two light cones with possibly different vertices in $\mathcal{M}^{4}$ are parallel in this sense.

Consider now two different compactifications of $\mathcal{M}^{4}: \overline{\mathcal{M}}_{p}^{4}$ and $\overline{\mathcal{M}}_{q}^{4}$.

$\overline{\mathcal{M}}_{p}^{4}$ and the future boundary. By definition, $\overline{\mathcal{M}}_{p}^{4}$ is the 4-dimensional real projective space $\mathbb{P}^{4}(\mathbb{R})$ which consists of $\mathcal{M}^{4}$ and points at infinity: one point at infinity corresponds to the full set of pairwise parallel lines in $\mathcal{M}^{4}$. Thus, in $\overline{\mathcal{M}}_{p}^{4}$ the Minkowski space-time $\mathcal{M}^{4}$ is compactified by a 3 -dimensional real projective space $\mathbb{P}^{3}(\mathbb{R})$. This boundary $\mathbb{P}^{3}(\mathbb{R})_{\infty}$ is endowed with an important additional structure, namely, an embedded 2-dimensional sphere $S^{2}$ which is the common base at infinity of all light cones of $\mathcal{M}^{4}$.

$\overline{\mathcal{M}}_{q}^{4}$ and the past boundary. We start with recalling that if $X$ is a smooth algebraic or analytic variety and $Y \subset X$ is a smooth closed subvariety, one can construct a morphism $b_{Y}: \widetilde{X} \rightarrow X$, in which $\widetilde{X}$ is another smooth variety, bl $Y_{Y}$ restricted to the complement $\widetilde{X} \backslash\left(\mathrm{bl}_{Y}\right)^{-1}(Y)$ defines its isomorphism with the initial complement $X \backslash Y$, whereas $\left(\mathrm{bl}_{Y}\right)^{-1}(Y)$ is the projectivized normal bundle to $Y$ in $X$, which $\mathrm{bl}_{Y}$ projects to its base $Y$.

In particular, if $\operatorname{dim} X=n$ and $Y$ is a point $x \in X$, then blowing it up, we get a divisor $\mathbb{P}^{n-1}$ which "squeezes into $X$ " replacing the former $x$. If we assume that $X$ is endowed with a conformal class of metrics, then in the tangent space to $x$ we have a canonically defined null cone, whereas in the projectivized tangent space embedded in the blow up it produces the "base" of the null cone, the local sky of the observer located at the point $x$ (see remark at p. 256 of [42] about the difference between the light cone which is a global object and the null cone which we invoked above).

This interpretation forms an essential part of the motivation for our constructions.

We will now describe $\overline{\mathcal{M}}_{q}^{4}$. By definition, it is a smooth real quadric hypersurface $Q^{4}$ in a five-dimensional projective space $\mathbb{P}^{5}(\mathbb{R})$, whose equation in homogeneous coordinates is given by a quadratic form of signature $(3,3)$. For any point $x \in Q^{4}$, one can construct the linear projective 4-dimensional subspace $\mathbb{P}_{x}^{4}$ in $\mathbb{P}^{5}(\mathbb{R})$ which is tangent to $Q^{4}$ at $x$. Then the intersection $\bar{L}_{x}:=Q^{4} \cap \mathbb{P}_{x}^{4}$ is isomorphic to any compactified light cone in $\overline{\mathcal{M}}_{p}^{4}$ above. Fix $x$ and consider the complement $Q^{4} \backslash L_{x}$. In this complement, through each point $y$ there passes the (uncompactified) light cone $L_{y}:=\bar{L}_{y} \backslash\left(\bar{L}_{y} \cap \bar{L}_{x}\right)$. One can identify $Q^{4} \backslash L_{x}$ with affine Minkovski space $\mathcal{M}^{4}$ by projecting $Q^{4} \backslash L_{x}$ from $x$ into any sufficiently general hyperplane $\mathbb{P}^{4}(\mathbb{R}) \subset \mathbb{P}^{5}(\mathbb{R})$.

More precisely, the same projection can be completed to a diagram of birational morphisms (restricted to real points of algebraic varieties defined over $\mathbb{R}$ )

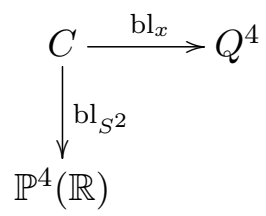

Namely, $C$ is obtained from $Q^{4}=\overline{\mathcal{M}}_{q}^{4}$ by blowing up the point $x \in Q^{4}$, and the same $C$ is obtained from $\mathbb{P}^{4}(\mathbb{R})=\overline{\mathcal{M}}_{p}^{4}$ by blowing up the infinite base of all light cones in $\mathbb{P}^{3}(\mathbb{R})=$ $\mathbb{P}^{4}(\mathbb{R}) \backslash M^{4}$.

\subsection{A warning: the orientation and topology of time}

For our present purposes, any time-like line in $\mathcal{M}^{4}$ may serve as a substitute of "time axis". It has the topology of the Euclidean line $\mathbb{R}$. 
In both compactifications that we have considered, $\overline{\mathcal{M}}_{p}^{4}$ and $\overline{\mathcal{M}}_{q}^{4}$, any time-like line has the topology of a circle $S^{1}$ : it is completed by just one point lying on the infinite hyperplane $\mathbb{P}^{3}(\mathbb{R})$, resp. $\mathbb{P}^{4}(\mathbb{R})$. Even if we orient this time circle, its infinite past coincides with its infinite future.

Time in this picture can be imagined as moving along a real projective line $\mathbb{P}^{1}(\mathbb{R})_{\text {time }}$. If we do not want to identify 0 with $\infty$, the beginning and the end of times, we must slightly change the definition of projective compactification of real space and the definition of real blow-up. This is done in Section 3 below. Briefly, physical time is oriented, because it flows irrevocably from past to future. Hence if we imagine a compactification of space-time compatible with the idea of physical time, it is natural to add to each time-like line two points: its "infinite past" $-\infty$ and "infinite future" $+\infty$. Mathematically, this leads to the consideration of the two-fold cover of the former $\mathbb{P}^{1}(\mathbb{R})_{\text {time. }}$. This cover topologically is still $S^{1}$, and the two points in each fiber of the cover correspond to two possible time orientations. Real blow-ups are defined similarly.

For the discussion below and in Section 4, it will be important to allow also "complex-valued time" and therefore to imagine $\mathbb{P}^{1}(\mathbb{R})_{\text {time }}$ embedded into $\mathbb{P}^{1}(\mathbb{C})_{\text {time }}$, the "complex projective line of time". Using a more sophisticated model of our Universe, namely, the Friedman-RobertsonWalker one, we will argue that $\mathbb{P}^{1}(\mathbb{C})_{\text {time }}$ naturally arises in it as the modular curve parametrizing elliptic curves.

In this case, we can imagine the time axis during one aeon modelled by the positive real semi-axis $[0,+\infty]$ in $\mathbb{P}^{1}(\mathbb{C})_{\text {time. }}$. This picture will allow us to appeal to some quantum ideas related to Wick's rotation, when time becomes purely imaginary.

\subsection{Penrose's cyclic cosmology}

Roger Penrose (see [42] and earlier publications) suggested that our observable Universe that started with the Big Bang was preceded by another stage ("aeon") of its development that ended as cold, infinite space (predicted to be the final stage of our Universe as well).

This idea seemingly implies the break of continuity between geometries of space-time during the transition between two aeons. The way Penrose suggested to overcome this break consisted in matching not the metrics of the respective space-times but the conformal classes of these metrics: he argued that rescaling the relevant Einstein metrics by conformal factors tending to zero, resp. infinity, one can avoid the apparent discrepancy: cf. a brief summary at pp. 204-205 of [42] and the first paragraph of [37].

We argue that Penrose's joining of two aeons can be modeled in our picture by identifying the future boundary of a previous aeon with the past (Big Bang) boundary of the next aeon, "crossover geometry".

As a model of the crossover geometry between $\overline{\mathcal{M}}_{p}^{4}$ and $\overline{\mathcal{M}}_{q}^{4}$ in our example, we suggest a choice from two possibilities.

Crossover model $I$. Identify the 3 -dimensional projective space $\mathrm{bl}_{x}^{-1}(x)$ with 3 -dimensional projective space at infinity of $\mathbb{P}^{4}(\mathbb{R})=\overline{\mathcal{M}}_{p}^{4}$ in such a way that the sphere of null-directions in $\mathrm{bl}_{x}^{-1}(x)$ is identified with the common base at infinity of all light cones in $\mathcal{M}^{4}$.

The space-time containing two aeons will consist of two irreducible components intersecting along the common crossover boundary $\mathbb{P}^{3}(\mathbb{R})$.

Crossover model II. In this model, the blowing up of the infinite $S^{2}$ is a model $C_{-}$of the first aeon preparing itself for the next Big Bang. The blowing up of $x$ is a model $C_{+}$of a Big Bang of the second aeon. Finally, the diagram (1.1) describes the geometry of matching the two aeons: it shows that the divisor $\mathrm{bl}_{S^{2}}^{-1}\left(\mathbb{P}^{3}(\mathbb{R})_{\infty}\right) \subset C_{-}$, "infinity of the first aeon", can be identified with the divisor $\mathrm{bl}_{x}^{-1}\left(L_{x}\right) \subset C_{+}$, the Big Bang of the next aeon, and after such an identification we get a connected space, say $C_{-} * C_{+}$, that can serve as a geometric model of the space-time including both aeons. 
The first crossover model is simpler and looks more universal. The choice between the two possibilities may be a matter of comparison with the geometry of the adopted differential geometric picture of the respective Einstein universes. There the structure of boundary is dictated by the considerations similar to those that led to the understanding of the Mixmaster model, see Section 4 below.

\subsection{Plan of the paper}

The next Section 2 is dedicated to the complexified space-times, involving spinors and Penrose's twistors. This context is convenient for introducing conformal classes of metrics in one framework with all necessary geometric tools. Moreover, basic physical fields, Lagrangians, and equations of motion (preceding quantisation) become very natural constructions: cf. Appendices in [42] and the survey [27]. In particular, we consider a complex version of the Big Bang diagram (1.1).

In the Section 3 we return to real models of space-time and discuss oriented versions of the diagram (1.1) and the respective notions of boundaries that arise in real algebraic geometry. They should be compared with more physical treatments: see in particular survey [15] and references therein. These considerations refer to what can be called "kinematic of boundaries".

Section 4 introduces an element of dynamic, namely the picture of time "on and near the boundary", or during and around the crossover.

After discussing the notion of cosmological time(s), the scheme we suggest is a formalisation of the intuitive idea that on the boundary "at the moment of Big Bang" time is purely imaginary. The complex projective line of physical time mathematically appears as the modular curve parametrizing the family of elliptic curves appearing in the description of Friedman-RobertsonWalker model, see Section 4.2 below. We suggest that the inverse Wick rotation needed to make time real is mediated by the evolution along a stretch of hyperbolic geodesic on the upper (or rather right, see Section 4.5) complex half-plane $H$ which is the standard cover of the respective modular curve. This allows us to include into our picture the chaos of early Mixmaster Universe, whose standard description involves exactly the same symbolic dynamics as that of hyperbolic geodesics, see Sections 4.3-4.5.

Notice that if we measure the cosmological time after Big Bang in terms of inverse temperature of the background radiation $1 / k T$, the backward passage to the imaginary time $i t / h$ generally transforms various partition functions of the chaos into their quantum versions, traces of evolving quantum operators.

If we want to use this picture for a description of crossover between two aeons, it remains only to assume that the real time of the previous aeon becomes imaginary at its future boundary.

We feel that here even our drastically simplified models must include elements of comparison with quantisation in order to describe what is going near the common boundary of two aeons. Traditionally, sufficiently symmetric models of space-time are quantized as Hamiltonian systems of classical mechanics (ADM quantisation). For a treatment of Bianchi models and Mixmaster solutions in this way see, e.g., [17] and [49]. For the relation to modular curves and modular symbols see $[29,30]$.

In the general algebraic spirit of this paper, the last Section 5 discusses options in the framework of $C^{*}$-quantisation formalisms. See also $[14,20]$ and references therein.

\section{A complex Big Bang model}

\section{$2.1 \quad$ Twistors and Grassmannian spinors}

The complex version of $\overline{\mathcal{M}}_{p}^{4}$ we consider is simply $\mathbb{P}^{4}$ (or rather $\mathbb{P}^{4}(\mathbb{C}$ ), but we will sometimes omit mentioning $\mathbb{C}$-points explicitly). 
The complex version of $\overline{\mathcal{M}}_{q}^{4}$ is the Grassmannian $\operatorname{Gr}(2, T)$ of 2-dimensional subspaces in the 4-dimensional complex vector space T, Penrose's twistor space.

Below we briefly recall the relevant geometric data. For more details, see [27, Chapter 1], in particular Section 3.

This Grassmannian carries the tautological vector bundle $S$ whose fiber over a point $x$ is the subspace $S(x) \subset T$ corresponding to this point. Moreover, we need the second spinor bundle $\widetilde{S}$, whose fiber $\widetilde{S}(x)$ over $x$ is the subspace orthogonal to $S(x)$ in the dual twistor space $T^{*}$. We use sometimes the respective sheaves of sections denoted $\widetilde{S}$ and $\widetilde{S}^{*}$ respectively.

The Grassmannian $\operatorname{Gr}(2, T)$ is canonically embedded into the projective space of lines in $\Lambda^{2} T$, $P\left(\Lambda^{2}(T)\right) \cong \mathbb{P}^{5}$, by the map $S \mapsto \Lambda^{2}(S)$ for each 2-dimensional subspace $S \subset T$. The image of this embedding $p$ is a 4-dimensional quadric hypersurface $G$ [27, Chapter 1, Section 3.2]. We have canonical isomorphisms

$$
\Lambda^{2}(S)=p^{*}\left(O_{P^{5}}(-1)\right), \quad \Lambda^{2}(\widetilde{S})=\Lambda^{2}(S) \otimes \Lambda^{4}(T)
$$

(see [27, Chapter 1, Section 1.4]).

\subsection{Tangent/cotangent bundles and light cones}

Moreover, we have canonical isomorphisms [27, Chapter 1, Section 1.6]

$$
\mathcal{T}_{G}=S^{*} \otimes \widetilde{S}^{*}, \quad \Omega_{G}^{1}=S \otimes \widetilde{S} .
$$

"Null" or "light" tangent vectors at a point $x$, by definition, correspond to the decomposable tangent directions $s^{*}(x) \otimes \tilde{s}^{*}(x)$ where $s^{*}(x) \in S^{*}(x), \tilde{s}^{*}(x) \in \widetilde{S}^{*}(x)$. A line in $G$ all of whose tangent vectors are null-vectors is called a light ray. Let $\mathbb{P}_{x}^{4}$ be the hyperplane in $\mathbb{P}\left(\Lambda^{2}(T)\right)$ tangent to $G$ at a point $x$. Then $\mathbb{P}_{x}^{4} \cap G$ is a singular quadric, the union of all light rays passing through $x$ in $G$, that is, complex light cone with vertex $x$. In view of (2.2), the base of this cone is canonically identified with $\mathbb{P}^{1}\left(S^{*}\right) \times \mathbb{P}^{1}\left(\widetilde{S}^{*}\right)$ [27, Chapter 1 , Section 3.6].

\subsection{Conformal metrics}

In this context, it is natural to define a conformal (class of) metric(s) as an invertible subsheaf of $S^{2}\left(\Omega^{1}\right)$ which is locally a direct summand. From (2.2) one sees that on $G$, we have such a subsheaf $\Lambda^{2}(S) \otimes \Lambda^{2}(\widetilde{S})$. A choice of the local section of $\Lambda^{2}(S) \otimes \Lambda^{2}(\widetilde{S})$ determines an actual (complex) metric wherever this section does not vanish.

Since $\Lambda^{2}(S) \otimes \Lambda^{2}(\widetilde{S}) \cong p^{*}\left(O_{P^{5}}(-2)\right)$ (see $(2.1)$ ), any such metric must have a pole on the intersection of $G$ with some quadric (possibly reducible, or even a double hyperplane) in $\mathbb{P}^{5}$, for example, a union of two light cones, that may even coincide. If we wish to compensate for this pole, we must locally multiply the metric by a meromorphic function vanishing at the polar locus. Imagining this polar locus as a "time horizon" of the Universe, we are thus bridging our picture with (real) conformal constructions by Penrose et al.

Finally, since for the definition and study of curved complex space-times the basic structure consists precisely of postulating two spinor bundles and an isomorphism (2.2) (see [41] and [27, Chapter 2]), essential features of the complexified blow up construction (1.1) can be generalized as we do below. We chose to describe only the local picture; it may become a part of a large spectrum of more global models.

\subsection{A complex blow up diagram}

Consider two complex four-dimensional manifolds $\overline{\mathcal{M}}_{-}$and $\overline{\mathcal{M}}_{+}$, non necessarily compact, with the following supplementary structures. 
(a) A smooth complex projective two-dimensional quadric $\mathcal{S}_{-} \cong \mathbb{P}^{1} \times \mathbb{P}^{1}$ embedded as a closed submanifold into $\overline{\mathcal{M}}_{-}$.

(b) A three-dimensional complex space $\mathcal{L}$ isomorphic to a neighborhood of the vertex of the complex cone with base $\mathbb{P}^{1} \times \mathbb{P}^{1}$ embedded as a closed submanifold into $\overline{\mathcal{M}}_{+}$.

If we blow up the vertex $x \in \overline{\mathcal{M}}_{+}$, the divisor $\mathbb{P}^{3}$ that replaces this vertex will contain the quadric $\mathcal{S}_{+}$of null-directions. Denote by $\widetilde{\mathcal{M}}_{+}$the result of such a blow up.

The last piece of the data we need is:

(c) An explicit isomorphism of $\mathcal{S}_{-}$in $\overline{\mathcal{M}}_{-}$with the quadrics of null directions $\mathcal{S}_{+}$in $\overline{\mathcal{M}}_{+}$.

These data will be (local, complex) analogs of $\overline{\mathcal{M}}_{p}^{4}$ endowed with heavens $S^{2}$, and of $\overline{\mathcal{M}}_{q}^{4}$ endowed with the light cone $L_{x}$ respectively, described in the Introduction.

Our complexified model of the transition between two aeons is then the connected sum $\overline{\mathcal{M}}_{-} * \mathcal{S} \widetilde{\mathcal{M}}_{+}$in which two complex quadrics $\mathcal{S}_{-}$and $\mathcal{S}_{+}$are identified.

\subsection{A multiverse model in twistor space}

We consider here a version of the blow-up and gluing construction carried out in twistor space, after composing with the Penrose twistor transform, and we relate the resulting "multiverse picture" to moduli spaces of configurations of trees of projective spaces recently introduced and studied from an algebraic geometric perspective in [7].

The Penrose transform is the correspondence given by the flag variety $F(1,2 ; T)$, with $T$ a complex 4-dimensional vector space, with projection maps to the complexified space-time given by the Grassmannian $\operatorname{Gr}(2 ; T)$ and to the twistor space given by the complex projective space $\mathbb{P}^{3}(\mathbb{C})$,

$$
\mathbb{P}^{3}=\operatorname{Gr}(1 ; T) \longleftarrow F(1,2 ; T) \longrightarrow \operatorname{Gr}(2 ; T) .
$$

We refer the reader to [27, Chapter 1, Section 4], for a more detailed exposition. The Penrose diagram (2.3) corresponds to the collection of $\alpha$-planes in the Klein quadric $\operatorname{Gr}(2 ; T) \hookrightarrow \mathbb{P}^{5}$. These planes, in which every line is a light ray, give one of the two families of planes corresponding to the two $\mathbb{P}^{1}$ 's in the base of a light cone $C(x)$. The planes in the $\alpha$-family are given by the second projection of the fibers of the first projection in (2.3). The other family of planes, the $\beta$-planes, are obtained similarly from the dual Penrose diagram

$$
\operatorname{Gr}\left(3 ; T^{*}\right) \longleftarrow F\left(2,3 ; T^{*}\right) \longrightarrow \operatorname{Gr}(2 ; T) .
$$

\subsection{Pointed rooted trees of projective spaces}

We consider oriented rooted trees that are finite trees with one outgoing flag (half-edge) at the root vertex and a number of incoming half-edges, with the tree oriented from the inputs to the root. Each vertex $v$ in the oriented rooted tree has one outgoing flag and $\operatorname{val}(v)-1$ incoming flags, each oriented edge $e$ in the tree is obtained as the matching of the unique outgoing flag at the source vertex with one of the incoming flags at the target vertex.

A rooted tree of projective spaces $\mathbb{P}^{d}$ is a rooted tree $\tau$ as above with a projective space $X_{v}=\mathbb{P}^{d}$ assigned to each vertex $v$, with a choice of a hyperplane $H_{v} \subset X_{v}$, and of a point $p_{v, f} \in X_{v}$ for each incoming flag $f$ at $v$, so that $p_{v, f} \neq p_{v, f^{\prime}}$ for $f \neq f^{\prime}$ and $p_{v, f} \notin H_{v}$ for all $f$. For each edge $e$ in the rooted tree $\tau$ we consider the blowup of the projective space $X_{t(e)}$ of the target vertex at the point $p_{t(e), f_{e}}$, and we glue the exceptional divisor $E_{t(e)}$ of the blowup $\mathrm{bl}_{p_{t(e), f e}}\left(X_{t(e)}\right)$ to the hyperplane $H_{s(e)} \subset X_{s(e)}$. When all these blowups and identifications are carried out, for all edges of $\tau$, one obtains a variety $X_{\tau}$, is a pointed rooted tree of projective spaces.

We consider the case where $d=3$. Each $\mathbb{P}^{3}$ in a tree of pointed projective spaces can be though of as the twistor space of a 4-dimensional complex spacetime. 
A hyperplane $H$ in the twistor space $\mathbb{P}^{3}$ corresponds, under the Penrose transform, to a $\beta$ plane in the Klein quadric, and points in $\mathbb{P}^{3}$ correspond to $\alpha$-planes. Thus, the choice of a hyperplane $H_{v} \subset X_{v}$ corresponds to fixing a $\beta$-plane in each copy $Q_{v}$ of the Klein quadric, while the choice of distinct points $p_{v, f}$ in $X_{v}$ not on the hyperplanes corresponds to a choice of $\alpha$-planes that do not meet the chosen $\beta$-plane.

We then come to a different model of gluing than the one discussed earlier, where the gluing is performed by blowing up the twistor spaces $X_{v}$ at the marked points $p_{v, f_{e}}$ of incoming flags $f_{e}$ corresponding to oriented edges $e$ of $\tau$ with $v=t(e)$ and gluing the exceptional divisor of the blowup to the hyperplane $H_{s(e)}$ in the twistor space $X_{s(e)}$.

This sequence of blowups and gluings produces a variety $X_{\tau}$, which is not necessarily itself the twistor space of a smooth 4-dimensional space-time. However, one can proceed in a way similar to the method used in [13], where one considers a gluing of blowups of twistor spaces and then deforms it to a new smooth twistor space. In our setting, the variety $X_{\tau}$ defines a point in the moduli space $T_{3, n}$ of stable deformations $n$-pointed rooted trees of projective spaces of [7], see also [31]. A path in $T_{3, n}$ from this point to a point in the open stratum provides a deformation to a single smooth twistor space with marked points and a marked hyperplane.

We can therefore interpret the moduli space $T_{3, n}$ as a multiverse landscape and any natural class of functions on this moduli space as multiverse fields.

\section{Real models and orientation}

\subsection{Projective spaces of real oriented lines}

As we mentioned, the diagram (1.1) is obtained by constructing first the respective diagram of algebraic geometric blow-ups and then restricting it to real points (in an appropriate real structure). In this section, we will discuss more physical versions of (1.1) first, by passing to certain unfamified coverings of the involved manifolds and second, by introducing "cuts" of these coverings that may be compared to various boundaries considered by physicists: causal, conformal etc. (see $[15,19]$ and references therein).

We start with real projective spaces.

Since $\mathbb{P}^{1}(\mathbb{R})$ is topologically $S^{1}$, we have $\pi_{1}\left(\mathbb{P}^{1}(\mathbb{R})\right) \cong \mathbb{Z}$. However, for $n \geq 2$ we have $\pi_{1}\left(\mathbb{P}^{n}(\mathbb{R})\right) \cong \mathbb{Z}_{2}$, and the universal covering of $\mathbb{P}^{n}(\mathbb{R})$ topologically is a certain double cover $S^{n} \rightarrow \mathbb{P}^{n}(\mathbb{R})$.

A more algebraic picture is this. Having chosen real homogeneous coordinates in $\mathbb{P}^{n}$, we may identify $\mathbb{P}^{n}(\mathbb{R})$ with $\left(\mathbb{R}^{n+1} \backslash\{0\}\right) / \mathbb{R}^{*}$ where the multiplicative group of reals $\mathbb{R}^{*}$ acts by multiplying all homogeneous coordinates of a point by the same factor. Now put

$$
\mathbb{P}_{\text {or }}^{n}(\mathbb{R}):=\left(\mathbb{R}^{n+1} \backslash\{0\}\right) / \mathbb{R}_{+}^{*},
$$

where $\mathbb{R}_{+}^{*}$ is the subgroup of positive reals.

Then the tautological map $\mathbb{P}_{\text {or }}^{n}(\mathbb{R}) \rightarrow \mathbb{P}^{n}(\mathbb{R})$ is the universal cover for $n \geq 2$. However, we may and will use this map also for $n=1$ and even $n=0$ since $\mathbb{P}_{\text {or }}^{n}(\mathbb{R})$ obviously parametrizes oriented lines in $\mathbb{R}^{n+1}$ for all values of $n \geq 0$.

\subsection{Boundaries}

In the situation of the previous subsection, consider the complete flag in $\mathbb{R}^{n+1}:\{0\}=\mathbb{R}^{0} \subset$ $\mathbb{R}^{1} \subset \mathbb{R}^{2} \subset \cdots \subset \mathbb{R}^{n+1}$, where $\mathbb{R}^{m}$ is the span of the first $m$ vectors of the chosen basis.

Then we get a chain of embeddings $\mathbb{P}_{\text {or }}^{0}(\mathbb{R}) \subset \mathbb{P}_{\text {or }}^{1}(\mathbb{R}) \subset \cdots \subset \mathbb{P}_{\text {or }}^{n}(\mathbb{R})$. Each $\mathbb{P}_{\text {or }}^{m}(\mathbb{R})$ is embedded in the next $\mathbb{P}_{\text {or }}^{m+1}(\mathbb{R})$ as $m$-dimensional equator $S^{m}$ of a sphere $S^{m+1}$. This equator cuts $\mathbb{P}_{\text {or }}^{m+1}(\mathbb{R})$ into two open subsets, say, $\mathbb{P}_{ \pm}^{m+1}(\mathbb{R})$, each of which embeds as a big cell into $\mathbb{P}^{m+1}(\mathbb{R})$; the choice 
of one of the components corresponds to the choice of orientation. The same reasoning applies to the equator itself $\mathbb{P}_{\text {or }}^{m}(\mathbb{R})$; we may choose an open half of it, say $\mathbb{P}_{+}^{m}(\mathbb{R})$ and add it to $\mathbb{P}_{+}^{m}(\mathbb{R})$, etc. The resulting partial compactification of $\mathbb{P}_{+}^{n+1}(\mathbb{R})$ may be considered as a compactification of space-time by a "future" boundary.

In particular, if we identify the big cell in $\mathbb{P}^{4}(\mathbb{R})$ with Minkowski space, and the last homogeneous coordinate with oriented time coordinate, we can choose the future part of the equatorial $\mathbb{P}_{\text {or }}^{3}(\mathbb{R})$ as one where the future part of (any) light cone finally lands.

Lifting the diagram (1.1) in this way to the diagram of parts of oriented Grassmannians, we finally get the algebraic geometric picture reflecting time orientation.

\subsection{Grassmannians of real oriented subspaces}

Similarly, the Grassmannian of real oriented $d$-dimensional subspaces in $\mathbb{R}^{d+c}$ is the double cover of the space of real points of the relevant complex Grassmanian, and it is the universal cover, if $c d \geq 2$.

One can extend the previous treatment of the case $d=1$ using "matrix homogeneous coordinates" on Grassmannians as in [27, Chapter 1, Section 1.3]. We will omit the details.

\subsection{Real points of a complex Big Bang model}

Real structures of complex spaces endowed with spinor bundles and isomorphisms (2.2) are discussed in [39, 41, 42] and [27]. In the local context of Section 2.4 the relevant spaces of real points of the quadrics $\mathcal{S}_{ \pm}$must have topology of $S^{2}$. One can also imagine the identification of these two $S^{2}$ 's as projection of the cylinder $S^{2} \times \mathbb{R}$ smashing the light-like axis $\mathbb{R}$. In this way the transition phase between two aeons is modeled by a trip along all light lines starting at the boundary. As we argue in the next section, physical time along a light geodesic does not "stop" as is usually postulated, but takes purely imaginary values. This is an additional argument to try the same picture for the crossover time between aeons.

\section{Big Bang models and families of elliptic curves}

\subsection{Time in cosmology and modular curves}

The primary notion of time in relativistic models is local: basically, along each time-like oriented geodesic the differential of its time function $d t$ is $d s$ restricted to this geodesic, where $d s^{2}$ is the relevant Einstein metric. Formally applying this prescription, we have to recognise that even in a flat space-time, along space-like geodesics time becomes purely imaginary, whereas light-like geodesics, along which time "stays still", form a wall. The respective wall-crossing in the space of geodesics produces the Wick rotation of time, from real axis to the pure imaginary axis. Along any light-like geodesic, "real" time stops, however "pure imaginary time flow" makes perfect sense appearing, e.g., as a variable in wave-functions of photons.

Below we will describe a model in which time is imaginary at the past boundary of the universe (or future boundary of the previous aeon), but the reverse Wick rotation does not happen instantly. Instead, it includes the movement of time along a curve in the complex plane. However, an important feature of this picture is that local times are replaced by a version of cosmological, i.e. global time, say $\Theta_{-}$, resp. $\Theta_{+}$, for the previous, resp. next aeon.

A good example of observable global time is the inverse temperature $1 / k T$ of the cosmic microwave background (CMB) radiation. It is accepted that the current value of it measures the global age of our Universe starting from the time when it stopped to be opaque for light, about $38 \cdot 10^{4}$ years after the Big Bang. 
Another version involves measuring the redshift of observable galaxies, thus putting their current appearance on various cosmological time sections of our Universe, so that the scientific picture of the observable Universe bears an uncanny resemblance to Marcel Duchamp's classics of modernism "Nude descending a staircase".

As we briefly described in Section 1.4, for us more important is not a choice of a concrete parametrisation of physical time (although we will use it later) but the image of an oriented time curve in the compactified complex plane $\mathbb{P}^{1}(\mathbb{C})_{\text {time. }}$. On the mathematical side, $\mathbb{P}^{1}(\mathbb{C})_{\text {time }}$ will appear as a modular curve parametrizing elliptic curves) that emerge, e.g., in Robertson-Walker and Bianchi models of the previous aeon.

Another $\mathbb{P}^{1}(\mathbb{C})$, a "modular one", contains the complex half-plane $H$ upon which the modular group $\operatorname{PSL}(2, \mathbb{Z})$ acts. We use an identification of $\mathbb{P}^{1}(\mathbb{C})_{\text {time }}$ with $\Gamma \backslash H$ where $\Gamma$ is $\operatorname{PSL}(2, \mathbb{Z})$ or a finite index subgroup of it.

We will now imagine cosmological times $\Theta_{ \pm}$as certain coordinate functions along the time curve lifted to the modular plane. When the universe attains along the real axis the wall from the side of the previous aeon, $\Theta_{-}=\infty$, the time curve moves to the imaginary axis containing the same point $\Theta_{-}=\infty=i \infty$, and follows it, say, from $i \infty$ to $i \epsilon_{-}$.

The imaginary moment $i \epsilon_{-}$is the beginning of the Big Bang of the next aeon.

After wall-crossing, time moves along a hyperbolic geodesic in the direction of the real axis. Along this geodesic, time has non-trivial real and imaginary components. When it reaches the real axis at a point $\epsilon_{+}$, it becomes real time $\Theta_{+}$of the next aeon.

In fact, in this situation we should think about geodesics on the right complex half-plane of time: $-i H=\{z \in \mathbb{C} \mid \operatorname{Re} z>0\}$ : see Section 4.5 below.

Physical evolution of the universe along the stretch of the geodesic is in principle a quantum phenomenon, unlike the classical models of cosmic space-time that we use in order to describe the aeons outside of the transition region.

However, this idea of non-trivial reverse Wick rotation allows us to incorporate the picture of the Mixmaster (Bianchi IX) Universe as a statistical dynamics approximation to an unknown quantum fields (or strings) picture of the Big Bang. Moreover, in our context it appears to be compatible with the Penrose picture, although many mathematical details are still to be worked out.

\subsection{Friedman-Robertson-Walker (FRW) universe and elliptic curves}

Following [46] and [37], we describe a previous aeon universe as (the late stage) of the FRW model.

In this model, the space-time (during one aeon) can be represented as the direct product of a global time $t$-axis and a maximally symmetric three-dimensional space section with a metric of constant curvature $k$. We choose also a fixed time-like geodesic ("observer's history") along which the metric is $d t^{2}$, and coordinatize each space section at the time $t$ by the invariant distance $r$ from the observer and two natural angle coordinates $\theta, \phi$ on the sphere of radius $r$. By rescaling the radial coordinate, we may and will assume that the curvature constant $k$ takes one of three values: $k= \pm 1$ or 0 .

This rescaling produces the natural unit of length, when $k \neq 0$, and the respective unit of time is always chosen so that the speed of light is $c=1$.

The RW metric of signature $(1,3)$ is then given by the formula

$$
d s^{2}:=d t^{2}-R(t)^{2}\left[\frac{d r^{2}}{1-k r^{2}}+r^{2}\left(d \theta^{2}+\sin ^{2} \theta d \phi^{2}\right)\right]
$$

It might be convenient to replace $r$ in (4.1) by the third dimensionless "angle" coordinate 
$\chi:=r / R(t)$. Then (4.1) becomes

$$
d s^{2}:=d t^{2}-R(t)^{2}\left[d \chi^{2}+S_{k}^{2}(\chi)\left(d \theta^{2}+\sin ^{2} \theta d \phi^{2}\right)\right],
$$

where $S_{k}(\chi)=\sin \chi$ for $k=1, \chi$ for $k=0$, and $\sinh \chi$ for $k=-1$.

Dynamics in this model is described by one real function $R(t)$ : it increases from zero at the Big Bang of one aeon to infinity during this aeon which, after the imaginary axis/geodesic transition described above, becomes "almost zero time" of the next aeon.

We scale $R(t)$ by putting $R=1$ "now", as in [46]. Notations in [46] slightly differ from ours. In his formula for metric (2), $r$ is our $\chi$, and $f_{k}(r)$ is our $S_{k}(\chi)$.

This function is constrained by the Einstein-Friedman equations (here with cosmological constant $\Lambda=3$ ), which leads to the introduction of the elliptic curve given by the equation in the $(Y, R)$-plane

$$
Y^{2}=R^{4}+a R+b
$$

(see [46, equation (3)] and [37, equation (9)], where their $S$ is the same as our $R$ ).

Besides the proper time $t$, and the scale factor $R(t)$, global time may be measured by its conformal version $\tau$, which according to [46, formula (3)] may be given as the integral along a real curve on the elliptic curve (4.2):

$$
\tau \cong \int_{0}^{R(t)} \frac{d R}{Y}
$$

A physical interpretation of the coefficients $a, b$ as characterising matter and radiation sources in (4.2), for which we refer the reader to [38] and [46], shows that in principle $a, b$ also depend on time, although for asymptotic estimates, their values are usually fixed by current observations.

We close this subsection by the following qualitative summary:

In the FRW universe, the time evolution is essentially described by a real curve on an algebraic surface (4.2) which is a family of elliptic curves.

Universal families of elliptic curves are parametrized by modular curves, and in the next subsection we will see a family of elliptic curves naturally emerges in the description of a late stage of evolution of the FRW model. In a pure mathematical context, the reader is invited to compare our suggestion with the treatment of the Painlevé VI equation in [28] and the whole hierarchy of Painlevé equations in [45].

\subsection{Bianchi IX universe and the modular curve}

As a model of the universe of the next aeon emerging after the Big Bang we take here the Bianchi IX space-time, admitting $\mathrm{SO}(3)$-symmetry of its space-like sections. Its metric in appropriate coordinates takes the following form:

$$
d s^{2}=d t^{2}-a(t)^{2} d x^{2}-b(t)^{2} d y^{2}-c(t)^{2} d z^{2},
$$

where the coefficients $a(t), b(t), c(t)$ are called scale factors.

A family of such metrics satisfying Einstein equations is given by Kasner solutions,

$$
a(t)=t^{p_{1}}, \quad b(t)=t^{p_{2}}, \quad c(t)=t^{p_{3}}
$$

in which $p_{i}$ are points on the real algebraic curve

$$
\sum p_{i}=\sum p_{i}^{2}=1 .
$$

These metrics become singular at $t=0$ which is the Big Bang moment. 
Around 1970, V. Belinskii, I.M. Khalatnikov, E.M. Lifshitz and I.M. Lifshitz argued that almost every solution of the Einstein equations for (4.3) traced backwards in time $t \rightarrow+0$ can be approximately described by a sequence of points (4.5): see [25] for a later and more comprehensive study. The $n$-th point of this sequence begins the respective $n$-th Kasner era, at the end of which a jump to the next point occurs, see below.

A mathematically more careful treatment of this discovery in [3] has shown that this encoding is certainly applicable to another dynamical system which is defined on the boundary of a certain compactification of the phase space of this Bianchi IX model and in a sense is its limit.

What makes this dynamical system remarkable in our context is that the construction involves a nontrivial real blow up at the $t=0$, see details in [4]. The resulting boundary, that we suggest to identify with the wall between two aeons, is an attractor, it supports an array of fixed points and separatrices, and the jumps between separatrices which result from subtle instabilities account for jumps between successive Kasner's regimes, corresponding to different points of (4.4).

In what sense this picture approximates the actual trajectories, is a not quite trivial question: cf. the last three paragraphs of [25, Section 2], where it is explained that among these trajectories there can exist "anomalous" cases when the description in terms of Kasner eras does not make sense, but that they are, in a sense, infinitely rare. See also the recent critical discussion in [26].

The remaining part of this section is dedicated to three subject matters:

(a) a description of the BKLL encoding of trajectories of Bianchi IX boundary solutions by sequences of points of (4.5);

(b) a description of encoding of (most) geodesics with finite ends on the complex upper halfplane $H$ by a version of the continued fractions formalism and their projections to the modular curve $\operatorname{PSL}(2, \mathbb{Z}) \backslash H$;

(c) a suggestion that the appropriate identification of these two descriptions corresponds to the identification of two evolutions, involving imaginary/complex time on and around the wall between two aeons. In fact both aeons then contribute mathematically comparable pictures of the time curve traced on a family of elliptic curves.

\subsection{BKLL encoding of Kasner eras}

Consider a "typical" solution (trajectory) $\gamma$ of the Einstein equations for (4.3) as $t \rightarrow+0$. Introduce the local logarithmic time $\Omega$ along this trajectory with inverted orientation. Its differential is $d \Omega:=-\frac{d t}{a b c}$, and the time itself is counted from an arbitrary but fixed moment. Then $\Omega \rightarrow+\infty$ approximately as $-\log t$ as $t \rightarrow+0$, and we have the following picture (perhaps strictly applicable only to the boundary system referred to above, see [25] and [3]).

(i) As $\Omega \cong-\log t \rightarrow+\infty$, a "typical" solution $\gamma$ of the Einstein equations determines a sequence of infinitely increasing moments $\Omega_{0}<\Omega_{1}<\cdots<\Omega_{n}<\ldots$ and a sequence of irrational real numbers $u_{n} \in(1,+\infty), n=0,1,2, \ldots$

(ii) The time semi-interval $\left[\Omega_{n}, \Omega_{n+1}\right)$ is called the $n$-th Kasner era (for the trajectory $\gamma$ ). Within the $n$-th era, the evolution of $a, b, c$ is approximately described by several consecutive Kasner's formulas. Time intervals where scaling powers $\left(p_{i}\right)$ are (approximately) constant are called Kasner's cycles.

(iii) The evolution in the $n$-th era starts at time $\Omega_{n}$ with a certain value $u=u_{n}>1$ which determines respective scaling powers during the first cycle in their growing order

$$
p_{1}=-\frac{u}{1+u+u^{2}}, \quad p_{2}=\frac{1+u}{1+u+u^{2}}, \quad p_{3}=\frac{u(1+u)}{1+u+u^{2}} \text {. }
$$

The next cycles inside the same era start with values $u=u_{n}-1, u_{n}-2, \ldots$, and scaling powers (4.6) corresponding to these numbers. 
(iv) After $k_{n}:=\left[u_{n}\right]$ cycles inside the current era, a jump to the next era comes, with parameter

$$
u_{n+1}=\frac{1}{u_{n}-\left[u_{n}\right]} .
$$

This means that the natural encoding of all $\left(u_{n}\right)$ together is obtained by considering an irrational number $x>1$ together with its continued fraction decomposition

$$
x=k_{0}+\frac{1}{k_{1}+\frac{1}{k_{2}+\cdots}}:=\left[k_{0}, k_{1}, k_{2}, \ldots\right] .
$$

The time flow is modelled by the powers of the discrete shift

$$
\left[k_{0}, k_{1}, k_{2}, \ldots\right] \mapsto\left[0, k_{0}, k_{1}, k_{2}, \ldots\right], \quad x \mapsto \frac{1}{x}-\left[\frac{1}{x}\right] .
$$

Put $x_{n}=\left[k_{n}, k_{n+1}, \ldots\right]$.

(v) We compare the initial time-point $\Omega_{n+1}$ of the next era with $\Omega_{n}$ by introducing the additional parameter $\delta_{n}$ via

$$
\Omega_{n+1}=\left(1+\delta_{n} k_{n}\left(u_{n}+1 / x_{n}\right)\right) \Omega_{n} .
$$

Then the information about both sequences $(u, \Omega)$ simultaneously can be encoded by two numbers $(x, y) \in(0,1)^{2}$, and the time flow can be modelled by powers of the shift of two-sided sequences of natural numbers

$$
\left[\ldots, k_{-2}, k_{-1}, k_{0}, k_{1}, k_{2}, \ldots\right]
$$

or else

$$
(x, y) \mapsto\left(\frac{1}{x}-\left[\frac{1}{x}\right], \frac{1}{y+[1 / x]}\right) .
$$

where $y=\left[0, k_{0}, k_{-1}, k_{-2}, \ldots\right]$.

More precisely, if we put then $\eta_{n}=\left(1-\delta_{n}\right) / \delta_{n}, x_{n}=u_{n}-k_{n}$, we get the following recursion relation:

$$
\eta_{n+1} x_{n}=\frac{1}{k_{n}+\eta_{n} x_{n-1}} .
$$

This means that in terms of the variables $\left(x_{n}, y_{n}:=\eta_{n+1} x_{n}\right)$ the transition to the next era is described by the (almost everywhere) invertible operator acting upon $[0,1] \times[0,1]$,

$$
\widetilde{T}:(x, y) \mapsto\left(\frac{1}{x}-\left[\frac{1}{x}\right], \frac{1}{y+[1 / x]}\right),
$$

which is studied in [34] and [25].

(vi) The rearrangement of scaling factors $p_{i}^{(n)}(u)$ in the increasing order induces generally a non-identical permutation of the respective coefficients.

Namely, as $u$ diminishes by 1 , the old permutation is multiplied by $(12)(3)$ (see [25, formula (2.3)]). When the era finishes, the permutation (1)(23) occurs (this is [25, formula (2.2)]).

This means that during one era, the largest exponent decreases monotonically, and governs the same scale factor, $a, b$, or $c$ which we will call the leading one. Two other exponents oscillate between the remaining pair of scaling coefficients. The number of oscillations is about $k_{n}:=\left[u_{n}\right]$.

In order to keep track of the sequence of the leading scale factors as well, we should consider orbits of $\operatorname{PGL}(2, \mathbb{Z})$ acting upon $\mathbb{P}^{1}(\mathbb{Q}) \times \mathbb{P}$ where $\mathbb{P}$ can be naturally identified with $\mathbb{P}^{1}\left(\mathbb{F}_{2}\right)=$ $\{1,0, \infty\}$. More precisely, the fractional linear transformation $u \mapsto 1 / u$ that corresponds to the transition to the new era, introduces the permutation $(1)(23)$ of $\{1,0, \infty\}$, whereas the passage to a new cycle within one era is described by the transformation $u \mapsto u-1$ which induces the permutation $(12)(3)$, see [29]. 


\subsection{Symbolic dynamics of the geodesic flow on the modular surface}

Miraculously, the same map (4.7) describes an appropriate Poincaré return map for the geodesic flow on the modular surface $\mathbb{M}$ which is either $\operatorname{PSL}(2, \mathbb{Z}) \backslash H$, or $\Gamma_{0}(2) \backslash H$, if we wish to take into account Kasner cycles within each era as at the end of Section 4.4, (iii) above. The relevant Poincare section is essentially the lift of the imaginary semi-axis of $H$ to $\mathbb{M}$. For more details, see $[6,14,43]$. In our context, an explanation of this coincidence is given by postulating the return of cosmological time to its real values mediated by a stretch of a hyperbolic geodesic.

A warning is in order here: when we embed the real time $\tau$ curve using an invariant of the elliptic curve (4.2) in the previous aeon, and a real geodesic on $\mathbb{M}$ in the following aeon, during the transition period we should interpret the upper half-plane $H$, or rather its compactified version $H \cup \mathbb{P}^{1}(\mathbb{R})$ as having the standard complex coordinate $z=-i \tau$. Then the part of the imaginary half-axis of $H$ between $i$ and $i \infty$ projects onto a real closed curve in $\mathbb{M}$ which is now simply a particular Poincaré section, a device for encoding more interesting "chaotic" time geodesics. On the contrary, the "imaginary time axis" on the "wall" between aeons invoked in Section 4.1 above becomes now $\mathbb{P}^{1}(\mathbb{R})$, the real boundary of $H$.

Of course, the action of $\operatorname{PSL}(2, \mathbb{Z})$ upon $\mathbb{P}^{1}(\mathbb{R})$ is topologically bad, and one can see in it the basic source of stochasticity during the transition period.

\section{Conformal gluing and $C^{*}$-models}

\subsection{Twisted spectral triples and conformal factors}

The notion of twisted spectral triples was introduced by Connes and Moscovici (see [12]) in order to extend the spectral triple formalism of noncommutative Riemannian spin geometry to type III cases that arise in the geometry of foliations and in other settings (see [20] for some cases related to number theory and to Schottky uniformizations).

The prototype example discussed in [12], which is also the most relevant one for our present purposes, is coming from the behaviour of the Dirac operator under conformal changes of the metric. We review it here briefly for later use.

Let $M$ be a compact Riemannian spin manifold. It is well known that the Riemannian geometry of $M$ can be reconstructed from its canonical spectral triple $(A, H, D)=\left(C^{\infty}(M), L^{2}(M, S)\right.$, $D)$, where $D$ is the Dirac operator. Thus, the notion of Riemannian spin geometry can be extended to the noncommutative setting, via spectral triples, where an abstract data $(A, H, D)$ now consist of a (possibly noncommutative) involutive algebra $A$, a representation of $A$ by bounded operators on a Hilbert space $H$, and a (densely defined) self-adjoint operator $D$ on $H$ with compact resolvent, satisfying the compatibility condition: boundedness of all commutators $[D, a]$ with elements $a \in A$.

Let $(M, g)$ be a compact $n$-dimensional Riemannian spin manifold with Dirac operator $D$. Consider a conformal change of the metric $g^{\prime}=\Omega^{2} g$, where we write $\Omega=e^{-2 h}$, for a real valued function $h \in C^{\infty}(M)$. As was observed in [12], after identifying the Hilbert spaces of square integrable spinors via the map of [5] scaled by $e^{n h}$, the relevant Dirac operators become related by $D^{\prime}=e^{h} D e^{h}$.

Moreover, according to [12], if the algebra $A_{Y}$ becomes noncommutative, the Dirac operator $e^{h} D_{Y} e^{h}$ no longer has bounded commutators with elements of the algebra. In this case, the correct notion that replaces the bounded commutator condition is the twisted version of [12].

Namely, for $D_{Y}^{\prime}=e^{h} D_{Y} e^{h}$ one requires the twisted commutators

$$
D_{Y}^{\prime} a-\sigma(a) D_{Y}^{\prime}=e^{h}\left[D_{Y}, e^{h} a e^{-h}\right] e^{h}
$$

to be bounded for all $a \in A_{Y}$, where $\sigma(a):=e^{2 h} a e^{-2 h}$. This replaces the ordinary notion of a spectral triple with the notion of twisted spectral triple. 


\subsection{Time evolution and conformal factors}

The expression above for the twisted commutator suggests that, in the case of a noncommutative algebra $A_{Y}$, one can consider a time evolution determined by the conformal factor, with $h=$ $h^{*} \in A_{Y}$ and $t \in \mathbb{R}$,

$$
\sigma_{t}(a)=e^{-i t h} a e^{i t h}
$$

Thus, consider the case of a Riemannian 4-dimensional geometry that is locally a cylinder $X=Y \times I$ with a metric $g_{X}=d t^{2}+g_{Y, t}$, where $g_{Y, t}=\Omega^{2}(t) g_{Y}$, for a fixed $g_{Y}$ and $\Omega(t)=e^{-2 h t}$, for some fixed $h \in C^{\infty}\left(Y, \mathbb{R}_{+}^{*}\right)$. If the algebra $C(Y)$ admits a noncommutative deformation compatible with the metric, then the transformation $a \mapsto e^{h} a e^{-h}$ that arises from the twisted commutator with the conformally rescaled Dirac operator can be seen as the effect of an evolution in imaginary time, under an analytic continuation to imaginary time of the time evolution defined above.

\subsection{Noncompact and Minkowskian geometries}

The setting of spectral triples (and by extension twisted spectral triples) can be generalized to the non-compact case, as in [18], by replacing the compact resolvent condition for the Dirac operator by a local condition, namely requiring that $a(D-\lambda)^{-1}$ is compact, for some $a \in A$ and $\lambda \notin \operatorname{Spec}(D)$. See [18, Section 3] for more details on the properties of non-compact spectral triples.

Accommodating Minkowskian geometries within the setting of spectral triples is a more delicate issue, because the Lorentzian Dirac operator is no longer self-adjoint and it is not an elliptic operator. A commonly used approach replaces Hilbert spaces with Krein spaces [2]. The case of flat Lorentzian cylinders over tori and their isospectral noncommutative deformations was treated with these techniques in [47]. Noncommutative Minkowskian geometry and isospectral noncommutative deformations were also considered in a number theoretic setting in [32].

\subsection{Noncommutativity from isospectral and toric deformations}

A general procedure to obtain noncommutative deformations of a commutative algebra of functions, in a way that preserves the metric structure, is through the isospectral deformations of [11]. Assume that the compact Riemannian manifold $Y$ is endowed with an action $\alpha: T^{2} \rightarrow \operatorname{Isom}\left(Y, g_{Y}\right)$ of a torus $T^{2}=U(1) \times U(1)$ by isometries. One obtains then a noncommutative deformation $A_{Y, \theta}$ of the algebra of functions $A_{Y}=C(Y)$, depending on a real parameter $\theta$, by the following procedure. Given $f \in C(Y)$, in the representation on the Hilbert space $H_{Y}=L^{2}\left(Y, S_{Y}\right)$, one decomposes the operator $\pi(f) \in B\left(H_{Y}\right)$ into weighted components according to the action of $T^{2}, \alpha_{\tau}\left(\pi\left(f_{n, m}\right)\right)=e^{2 \pi i\left(n \tau_{1}+m \tau_{2}\right)} \pi\left(f_{n, m}\right)$ The deformed product is then given on components by $f_{n, m} \star_{\theta} h_{k . r}=e^{\pi i \theta(n r-m k)} f_{n, m} h_{k, r}$. Geometrically, this corresponds to deforming the torus $T^{2}$ to noncommutative torus $T_{\theta}^{2}$, where one defines the algebra of the noncommutative torus as the twisted group algebra $C^{*}\left(\mathbb{Z}^{2}, \gamma\right)$ with cocycle $\gamma((n, m),(k, r))=\exp (\pi i \theta(n r-m k))$. The deformation $A_{Y, \theta}$ is isospectral, in the sense that the data $\left(H_{Y}, D_{Y}\right)$ of the spinor space and Dirac operator, that determine the metric structure, remains undeformed. Theta deformations, for $\theta$ a skew-symmetric matrix, can similarly be obtained for compact Riemannian manifolds $Y$ with an isometric action of a higher dimensional torus $T^{k}=U(1)^{k}$, which is similarly deformed to a noncommutative torus $T_{\theta}^{k}$.

The construction of such $\theta$-deformations in the setting of noncommutative Riemannian geometry was extended to an algebro-geometric setting in the work of Cirio, Landi and Szabo [8, 9, 10], by replacing the real noncommutative tori with algebraic noncommutative tori. This leads to 
noncommutative deformations of projective spaces and other toric varieties, as well as deformations of Grassmannians, via a deformation of their Plücker coordinates.

\subsection{Noncommutative deformations of complex space-times}

Consider the complex 4-manifolds $\overline{\mathcal{M}}_{ \pm}$involved in the blowup diagram giving the gluing of successive aeons in our complex big bang model. As we have seen, these are, respectively, the projective space $\mathbb{P}^{4}$ and the Grassmannian $\operatorname{Gr}(2, T)$ of 2-planes in a 4-dimensional complex vector space $T$. We view the Grassmannian $\operatorname{Gr}(2, T)$ as embedded in $\mathbb{P}\left(\Lambda^{2} T\right) \simeq \mathbb{P}^{5}$ under the Plücker map, with image the Klein quadric $Q$ in $\mathbb{P}^{5}$.

The appropriate noncommutative $\theta$-deformation for the Klein quadric (and for more general Grassmannians) was constructed in $[8,9]$, in terms of homogeneous coordinates in $\theta$-deformed projective spaces and noncommutative Plücker relations.

More precisely, we have $\theta$-deformations $\mathbb{P}_{\theta}^{n}$ of projective spaces, whose homogeneous coordinate algebra has generators $\left\{w_{i}\right\}_{i=1, \ldots, n+1}$ and relations $w_{i} w_{j}=q_{i j}^{2} w_{j} w_{i}$, for $i, j=1, \ldots, n$, with $q_{a b}=\exp \left(\frac{i}{2} \theta_{a b}\right)$ and $w_{n+1} w_{i}=w_{i} w_{n+1}$, for $i=1, \ldots, n$.

The algebra of functions on the noncommutative Grassmannian $\operatorname{Gr}_{\theta}(2, T)$ has six generators, $\left\{\Lambda^{I}=\Lambda^{(i j)}\right\}_{1 \leq i<j \leq 4}$, labeled by minors $I$ of a $2 \times 4$-matrix. In general, for a Grassmannian $\operatorname{Gr}_{\theta}(d ; n)$ these variables $\Lambda^{J}$, for minors $J=\left(j_{1}, \ldots, j_{d}\right)$, satisfy relations

$$
\Lambda^{J} \Lambda^{J^{\prime}}=\left(\prod_{\alpha, \beta=1}^{d} q_{j_{\alpha}, j_{\beta}^{\prime}}^{2}\right) \Lambda^{J^{\prime}} \Lambda^{J},
$$

as in [10, equation (1.26)].

The skew-symmetric matrix $\Theta$ is related to $\theta$ as in [10, equation (1.28)]

$$
\Theta^{J J^{\prime}}=\sum_{\alpha, \beta=1}^{d} \theta^{j_{\alpha} j_{\beta}^{\prime}},
$$

as a necessary and sufficient condition for the existence of Plücker embedding.

The noncommutative Plücker embedding of the deformed $\operatorname{Gr}_{\theta}(2, T)$ in $\mathbb{P}_{\Theta}^{5}$ is then determined by the relation

$$
q_{31} q_{32} q_{34} \Lambda^{(12)} \Lambda^{(34)}-q_{21} q_{23} q_{24} \Lambda^{(13)} \Lambda^{(24)}+q_{12} q_{13} q_{14} \Lambda^{(23)} \Lambda^{(14)}=0,
$$

where $q_{i j}$ were defined above.

It is also shown in [10] that there is a compatible real structure on the deformed Grassmannian $\operatorname{Gr}_{\theta}(2, T)$ and a unique $\theta$-deformation of the sphere $S^{4}$ that is compatible with a noncommutative twistor correspondence. As shown in [10, Section 2.3], this corresponds to the involutive $\star-$ algebra structure on the noncommutative Klein quadric for which $q_{12}=q_{21}^{-1}=q$ and the other $q_{i j}=1$.

The standard construction of Schubert cells is also compatible with this quantization.

The Grassmannian $\operatorname{Gr}(2, T)$, which gives the complexified spacetime, has a cell decomposition into six Schubert cells $C_{\left(j_{1}, j_{2}\right)}$, with

$$
\left(j_{1}, j_{2}\right) \in\{(1,2),(1,3),(1,4),(2,3),(2,4),(3,4)\},
$$

respectively of complex dimensions $0,1,2,2,3,4$. They correspond to $2 \times 4$-matrices in row echelon form, and consist of 2-planes $V$ that intersect the standard flag $F$ with $\operatorname{dim}\left(V \cap F_{j_{\ell}}\right)=\ell$. In terms of the Plücker embedding $\operatorname{Gr}(2, T) \hookrightarrow \mathbb{P}^{5}$, if we write the defining equation for $\operatorname{Gr}(2, T)$ in $\mathbb{P}^{5}$ as above, in the form

$$
\Lambda^{(12)} \Lambda^{(34)}-\Lambda^{(13)} \Lambda^{(24)}+\Lambda^{(23)} \Lambda^{(14)}=0,
$$


then the Schubert varieties $X_{\left(j_{1}, j_{2}\right)}$ given by the closures of the Schubert cells, $X_{\left(j_{1}, j_{2}\right)}=\overline{C_{\left(j_{1}, j_{2}\right)}}$, are given, respectively, by equations

$$
\begin{aligned}
& X_{(1,2)}=\left\{V \in \operatorname{Gr}(2, T) \mid \Lambda^{(13)}=\Lambda^{(14)}=\Lambda^{(23)}=\Lambda^{(24)}=\Lambda^{(34)}=0\right\}, \\
& X_{(1,3)}=\left\{V \in \operatorname{Gr}(2, T) \mid \Lambda^{(14)}=\Lambda^{(23)}=\Lambda^{(24)}=\Lambda^{(34)}=0\right\}, \\
& X_{(1,4)}=\left\{V \in \operatorname{Gr}(2, T) \mid \Lambda^{(23)}=\Lambda^{(24)}=\Lambda^{(34)}=0\right\}, \\
& X_{(2,3)}=\left\{V \in \operatorname{Gr}(2, T) \mid \Lambda^{(14)}=\Lambda^{(24)}=\Lambda^{(34)}=0\right\}, \\
& X_{(2,4)}=\left\{V \in \operatorname{Gr}(2, T) \mid \Lambda^{(34)}=0\right\},
\end{aligned}
$$

with $X_{(3,4)}=\operatorname{Gr}(2, T)$.

\subsection{Quantization and gluing of aeons}

By the explicit description of Schubert cells and Schubert varieties that we recalled above, we see that the quantization $\operatorname{Gr}_{\theta}(2, T)$, given by deforming the Plücker embedding to

$$
q_{31} q_{32} q_{34} \Lambda^{(12)} \Lambda^{(34)}-q_{21} q_{23} q_{24} \Lambda^{(13)} \Lambda^{(24)}+q_{12} q_{13} q_{14} \Lambda^{(23)} \Lambda^{(14)}=0
$$

induces compatible quantizations of the Schubert varieties $X_{\left(j_{1}, j_{2}\right)}$. In particular, for the closure $X_{(2,4)}$ of the 3 -dimensional cell, with the set of quantization parameters given by the $q_{a b}$ of $\operatorname{Gr}_{\theta}(2, T)$ with $a \neq 3$.

When we view $\operatorname{Gr}(2, T)$ as complex spacetime, the big cell $U=C_{(3,4)}$ is the complexified Minkowski space and the Schubert variety $X_{(2,4)}$ can be identified with the boundary $C(\infty)$ given by light rays through infinity, see [27, Chapter 1, Section 3.9]. The description of the cone $C(\infty)$ as the locus of $V \in \operatorname{Gr}(2, T)$ with $\operatorname{dim}\left(V \cap F_{2}\right) \geq 1$, with respect to the chosen flag $F$, corresponds to the usual description of the codimension one Schubert cycle in $\operatorname{Gr}(2, T)$.

Let us then consider again, in these terms, the two Crossover models from Section 1.3. To make the case of Crossover model I compatible with the $\theta$-deformations, it suffices to use a $\theta$ deformation of the exceptional divisor $\mathbb{P}^{3}$ of the blowup of $Q^{4}$ where the deformation parameters match the deformation parameters of the $\mathbb{P}^{3}$ at infinity of $\mathbb{P}^{4}$.

We consider then the case of Crossover model II. It is based upon identification of the intersection $\bar{L}_{x}=Q^{4} \cap \mathbb{P}_{x}^{4}$ in $\mathbb{P}^{5}$ and a compactified light cone in $\overline{\mathcal{M}}_{p}^{4}$, which leads to the blowup diagram of the transition between aeons. We check that the picture still makes sense when we pass to compatible $\theta$-deformations.

In the case of $\overline{\mathcal{M}}_{q}^{4}$, the Klein quadric $Q^{4}$ in $\mathbb{P}^{5}$, we use the quantization of [9] described above, with the compatible quantization on the Schubert varieties $X_{\left(j_{1}, j_{2}\right)}$. In the case of $\overline{\mathcal{M}}_{p}^{4}=\mathbb{P}^{4}(\mathbb{C})$ we consider a ruled surface defined by the equation $x_{0} x_{3}+x_{1} x_{2}=0$ inside the $\mathbb{P}^{3}$ defined by setting $x_{4}=0$. This can be seen as the intersection of the Klein quadric with the $\mathbb{P}^{3}$ given by $x_{4}=0$ and $x_{5}=0$ in $\mathbb{P}^{5}$. Thus, we can compatibly quantize the $\mathbb{P}^{4}(\mathbb{C})$ and the hyperplane $\mathbb{P}^{3}$ cut out by $x_{4}=0$ and the locus $x_{0} x_{3}+x_{1} x_{2}=0$ using the same quantization parameters of that we used for $\mathbb{P}^{5}$ and the Klein quadric $Q^{4}$ in it.

\subsection{Conformal cyclic cosmology versus Mixmaster universe}

Both Penrose's conformally cyclic cosmology model and the older Mixmaster universe model provide models of the universe undergoing a series of cycles, or aeons. However, the two models differ significantly in the way the cycling happens and in the physical properties that describe the behavior near the singularity. A comparative analysis of Mixmaster type models and conformally cyclic cosmology can be found in Sections 2.4, 2.6 and 3.1 of [42]. 
The main difference between these two models lies in the fact that conformally cyclic cosmology requires a conformally flat isotropic spacetime, while the spacetime of the Mixmaster universe is highly anisotropic. Moreover, conformally cyclic cosmology requires a vanishing or at least bounded Weyl curvature at the singularity, which is related to a very low entropy by Penrose's Weyl curvature hypothesis [40], while the Weyl curvature diverges widely in the Mixmaster case, with a high entropy singularity. Thus, one can consider the Mixmaster model as representing the generic, typical, conditions of the collapse, with high entropy and chaotic dynamics, and the conformally cyclic cosmology as a very special low entropy scenario.

The algebraic geometric setting we consider here can be adapted to accommodate both the conformally cyclic cosmology to a geometry and a Mixmaster type model. We have seen how to describe the conformal gluing of eons in terms of blowup diagrams. We now consider a similar picture for a Mixmaster type cosmology.

Let us consider again the case of a ruled quadric $\mathcal{S} \simeq \mathbb{P}^{1} \times \mathbb{P}^{1}$ in a hyperplane $\mathbb{P}^{3} \subset \mathbb{P}^{4}$ and the blowup $\operatorname{bl}_{\mathcal{S}}\left(\mathbb{P}^{4}\right)$. The exceptional divisor $E$ of the blowup is a projective bundle over $\mathcal{S}$ with fiber $\mathbb{P}^{\operatorname{codim}(\mathcal{S})-1}=\mathbb{P}^{1}$.

In the case of conformally cyclic cosmology, we considered the real structure as in [27, Chapter 1, Sections 3.9-3.19], where the real involution on $\mathcal{S}$ comes from exchanging the two spinor bundles $S$ and $\tilde{S}$, so that $S(\mathbb{R})=S^{2}$, the diagonal $\mathbb{P}^{1}(\mathbb{C})$, interpreted as the "sky". We now consider instead a different real structure where the real involution is the standard conjugation on each of the two copies of $\mathbb{P}^{1}$, which gives a set of real points $\mathcal{S}(\mathbb{R})=S^{1} \times S^{1}$, given by the two $\mathbb{P}^{1}(\mathbb{R})$. In this model, the real $E(\mathbb{R})$ has (locally) a product structure as a circle-bundle over $T^{2}=S^{1} \times S^{1}$. The gluing of successive aeons now happens along this circle-bundle.

In this case we restrict to considering metrics that are compatible with the local product structure on the exceptional divisor $E$ in the blowup, so that the metric restricted to $E$ has the diagonal form $h_{1}\left(z_{1}\right)+h_{2}\left(z_{2}\right)+h_{3}\left(z_{3}\right)$ where each $h_{i}$ is a Riemannian metric on $\mathbb{P}^{1}$. We identify the gluing region locally as a one parameter family with fiber $E$ over a line (representing the complexified time coordinate). Then, instead of modifying the metric as in the conformally cyclic cosmology, by multiplying by a single meromorphic function vanishing at infinity, we can now consider non-isotropic metrics adapted to the product structure, of the form $a(z)^{2} h_{1}\left(z_{1}\right)+b(z)^{2} h_{2}\left(z_{2}\right)+c(z)^{2} h_{3}\left(z_{3}\right)$, with $z$ the complexified time coordinate and, in particular, metrics where the factors $a(z), b(z), c(z)$ are of Kasner form $a(z)=z^{p_{1}}, b(z)=z^{p_{2}}$

and $c(z)=z^{p_{3}}$ with $\sum_{i} p_{i}=\sum_{i} p_{i}^{2}=1$, where the exponents $p_{i}$ are chosen, in each Kasner era, according to the prescription described in Sections 4.4 and 4.5, when the complex time coordinate moves along geodesics in the modular $\mathbb{P}^{1}$. We'll see below that this embedding of Mixmaster type cosmologies inside the complex blowup diagram of cyclic cosmology leads to a compatible quantization picture.

\subsection{Comparing quantizations}

We can also compare the quantization by $\theta$-deformation of the conformally cyclic cosmology model described above with the quantization by $\theta$-deformation of the Mixmaster universe described in [14]. To obtain a $\theta$-deformation of the gluing region in the Mixmaster type model described above, we consider an algebraic $\theta$-deformation (in the sense of $[8,9]$ ) of the $\mathbb{P}^{1}$-bundle $E$ over $\mathcal{S}=\mathbb{P}^{1} \times \mathbb{P}^{1}$, which induces a $C^{*}$-algebraic $\theta$-deformation on the circle bundle $E(\mathbb{R})$ over $T^{2}$, locally modelled on a noncommutative 3 -torus $T_{\theta}^{3}$. This is compatible with the $\theta$-deformations of the Mixmaster universe cosmology considered in [14] based on noncommutative 3-tori. Notice that, if we start from a $\theta$-deformation of the ambient complex spacetime $\mathbb{P}^{4}$ (or $\operatorname{Gr}(2, T)$ ) which induces a compatible $\theta$-deformation of $\mathcal{S}$ (see the discussion above about the cells of the Grassmannian), then a compatible deformation of $E$ will maintain the fiber direction as a distinct (commutative) direction, while if we only require the quantization to happen at (or 
near) the gluing, then more general $\theta$-deformations of $E$ can be considered, analogous to the noncommutative 3 -tori of [14]. The fact that one of the three spatial directions is singled out is compatible with the fact that the Mixmaster models have, in each Kasner era, a distinguished spatial direction that dominates the expansion or contraction.

\subsection{Predictive aspects}

In this paper we are primarily concerned with the development of mathematical models for various aspects of cosmology, based on the use of algebro-geometric techniques and noncommutative deformations.

At this stage of development of these models, it is premature to focus on predictions that may be detected in observational cosmology. However, we point out some directions in which one may be able to explore more concrete predictive aspects, though these would first require further theoretical developments.

It is well known that Penrose's cyclic cosmology model ran into serious difficulties in the verification of predictions on signatures of ripples from previous aeons in the background radiation. In particular, the evidence proposed in [22] was seriously criticized in [23, 48], and [35], see also the answer of [21]. At the theoretical level, Penrose's model is also criticized for the lack of a specific physical process that causes the described transition between aeons (unlike the dynamical fields responsible for a similar behavior in the Steinhardt-Turok cyclic universe). As observed in [46], a bound on the angular size of the circles hypothesized by Penrose (as would follow from [22] and [21]) would not be compatible with inflation in the early universe.

The theory of inflationary cosmology recently received a spectacular observational verification, along with primordial gravitational waves, announced by the BICEP2 team [1]. However, these findings are also currently being scrutinized for possible cosmic dust contributions to the signal, see [50], awaiting an independent analysis based on the Planck data, presently still to be released.

The multiverse picture presented in Section 2.5 fits more closely within the inflationary models than Penrose's original cyclic cosmology scenario. In particular, it may be related to the type of eternal inflation scenarios developed, in a discretized model, in [24]. Thus, possible predictions may be obtainable in this setting by developing a more detailed analysis of the "multiverse fields" outlined in Section 2.5, as in [24]. Algebro-geometric methods in the context of [24] have already been considered in [33].

In a different direction, one can seek predictions with possible observational verification, pertaining to the quantization picture described in Section 5. This is based on noncommutative deformations of spacetime near the singularity. There has been considerable work in the past on obtaining predictions of signals of early universe noncommutativity that would be detectable in the CMB sky, see for instance $[16,36,44]$. The question, in this case, would be to identify specific signatures of the type of noncommutative deformation considered here, based on the $\theta$-deformation method.

\section{Acknowledgements}

This paper was conceived after the lecture in Bonn (November 2013), in which Sir Roger Penrose explained his fascinating ideas about cyclic cosmology. Ya. Sinai and O. Bogoyavlenskii made helpful remarks about BKLL treatment of the Bianchi IX model. We are grateful to them.

\section{References}

[1] BICEP2 Collaboration, BICEP2 I: Detection of B-mode polarization at degree angular scales, Phys. Rev. Lett. 112 (2014), 241101, 25 pages, arXiv:1403.3985. 
[2] Bognár J., Indefinite inner product spaces, Springer-Verlag, New York - Heidelberg, 1974.

[3] Bogoyavlenskiu O.I., Novikov S.P., Singularities of the cosmological model of the Bianchi $I X$ type according to the qualitative theory of differential equations, J. Exp. Theor. Phys. 64 (1973), 1475-1494.

[4] Bogoyavlensky O.I., Methods in the qualitative theory of dynamical systems in astrophysics and gas dynamics, Springer Series in Soviet Mathematics, Springer-Verlag, Berlin, 1985.

[5] Bourguignon J.-P., Gauduchon P., Spineurs, opérateurs de Dirac et variations de métriques, Comm. Math. Phys. 144 (1992), 581-599.

[6] Chang C.-H., Mayer D., Thermodynamic formalism and Selberg's zeta function for modular groups, Regul. Chaotic Dyn. 5 (2000), 281-312.

[7] Chen L., Gibney A., Krashen D., Pointed trees of projective spaces, J. Algebraic Geom. 18 (2009), 477-509, math.AG/0505296.

[8] Cirio L., Landi G., Szabo R.J., Algebraic deformations of toric varieties. II. Noncommutative instantons, Adv. Theor. Math. Phys. 15 (2011), 1817-1907, arXiv:1106.5708.

[9] Cirio L.S., Landi G., Szabo R.J., Algebraic deformations of toric varieties. I. General constructions, Adv. Math. 246 (2013), 33-88, arXiv:1001.1242.

[10] Cirio L., Landi G., Szabo R.J., Instantons and vortices on noncommutative toric varieties, arXiv:1212.3469.

[11] Connes A., Landi G., Noncommutative manifolds, the instanton algebra and isospectral deformations, Comm. Math. Phys. 221 (2001), 141-159, math.QA/0011194.

[12] Connes A., Moscovici H., Type III and spectral triples, in Traces in number theory, geometry and quantum fields, Aspects Math., Vol. E38, Friedr. Vieweg, Wiesbaden, 2008, 57-71, math.OA/0609703.

[13] Donaldson S., Friedman R., Connected sums of self-dual manifolds and deformations of singular spaces, Nonlinearity 2 (1989), 197-239.

[14] Estrada C., Marcolli M., Noncommutative Mixmaster cosmologies, Int. J. Geom. Methods Mod. Phys. 10 (2013), 1250086, 28 pages, arXiv:1203.2679.

[15] Flores J.L., Herrera J., Sánchez M., On the final definition of the causal boundary and its relation with the conformal boundary, Adv. Theor. Math. Phys. 15 (2011), 991-1057, arXiv:1001.3270.

[16] Fukuma M., Kono Y., Miwa A., Effects of space-time noncommutativity on the angular power spectrum of the CMB, Nuclear Phys. B 682 (2004), 377-390, hep-th/0307029.

[17] Furusawa T., Quantum chaos of Mixmaster universe, Progr. Theoret. Phys. 75 (1986), 59-67.

[18] Gayral V., Gracia-Bondía J.M., Iochum B., Schücker T., Várilly J.C., Moyal planes are spectral triples, Comm. Math. Phys. 246 (2004), 569-623, hep-th/0307241.

[19] Geroch R., Kronheimer E.H., Penrose R., Ideal points in space-time, Proc. Roy. Soc. London Ser. A 327 (1972), 545-567.

[20] Greenfield M., Marcolli M., Teh K., Twisted spectral triples and quantum statistical mechanical systems, arXiv:1305.5492.

[21] Gurzadyan V.G., Penrose R., More on the low variance circles in CMB sky, arXiv:1012.1486.

[22] Gurzadyan V.G., Penrose R., On CCC-predicted concentric low-variance circles in the CMB sky, Eur. Phys. J. 128 (2013), 22, 17 pages, arXiv:1302.5162.

[23] Hajian A., Are there echoes from the Pre-Big Bang Universe? A search for low variance circles in the CMB sky, Astrophys. J. 740 (2011), no. 2, 52, 4 pages, arXiv:1012.1656.

[24] Harlow D., Shenker S.H., Stanford D., Susskind L., Tree-like structure of eternal inflation: a solvable model, Phys. Rev. D 85 (2012), 063516, 24 pages, arXiv:1110.0496.

[25] Khalatnikov I.M., Lifshitz E.M., Khanin K.M., Shchur L.N., Sină̌ Y.G., On the stochasticity in relativistic cosmology, J. Statist. Phys. 38 (1985), 97-114.

[26] Lugo L.M., Chauvet P.A., BKL method in the Bianchi IX universe model revisited, Appl. Phys. Res. 5 (2013), no. 6, 107-117.

[27] Manin Yu.I., Gauge field theory and complex geometry, Grundlehren der Mathematischen Wissenschaften, Vol. 289, 2nd ed., Springer-Verlag, Berlin, 1997.

[28] Manin Yu.I., Sixth Painlevé equation, universal elliptic curve, and mirror of $\mathbb{P}^{2}$, in Geometry of Differential Equations, Amer. Math. Soc. Transl. Ser. 2, Vol. 186, Editors A. Khovanskii, A. Varchenko, V. Vassiliev, Amer. Math. Soc., Providence, RI, 1998, 131-151, alg-geom/9605010. 
[29] Manin Yu.I., Marcolli M., Continued fractions, modular symbols, and noncommutative geometry, Selecta Math. (N.S.) 8 (2002), 475-521, math.NT/0102006.

[30] Manin Yu.I., Marcolli M., Modular shadows and the Lévy-Mellin $\infty$-adic transform, in Modular Forms on Schiermonnikoog, Editors B. Edixhoven, G. van der Geer, B. Moonen, Cambridge University Press, Cambridge, 2008, 189-238, math.NT/0703718.

[31] Manin Yu.I., Marcolli M., Moduli operad over $\mathbb{F}_{1}$, in Absolute Arithmetic and $\mathbb{F}_{1}$ Geometry, Editor K. Thas, Eur. Math. Soc., 2013, 331-364, arXiv:1302.6526.

[32] Marcolli M., Solvmanifolds and noncommutative tori with real multiplication, Commun. Number Theory Phys. 2 (2008), 421-476, arXiv:0711.2036.

[33] Marcolli M., Tedeschi N., Multifractals, Mumford curves and eternal inflation, p-Adic Numbers Ultrametric Anal. Appl. 6 (2014), 135-154, arXiv:1311.5458.

[34] Mayer D.H., Relaxation properties of the Mixmaster universe, Phys. Lett. A 121 (1987), 390-394.

[35] Moss A., Scott D., Zibin J.P., No evidence for anomalously low variance circles on the sky, J. Cosmol. Astropart. Phys. 2011 (2011), no. 4, 033, 7 pages, arXiv:1012.1305.

[36] Nautiyal A., Anisotropic non-gaussianity with noncommutative spacetime, Phys. Lett. B 728 (2014), 472481, arXiv:1303.4159.

[37] Newman E.T., A fundamental solution to the CCC equations, Gen. Relativity Gravitation 46 (2014), no. 5, 1717, 13 pages, arXiv:1309.7271.

[38] Olive K.A., Peacock J.A., Big-Bang cosmology, available at http://pdg.1bl.gov/2005/reviews/ bigbangrpp.pdf.

[39] Penrose R., Conformal treatment of infinity, in Relativité, Groupes et Topologie (Lectures, Les Houches, 1963 Summer School of Theoret. Phys., Univ. Grenoble), Editors B. deWitt, C. deWitt, Gordon and Breach, New York, 1964, 565-584.

[40] Penrose R., Singularities and time-asymmetry, in General Relativity: an Einstein Centenary Survey, Editors S.W. Hawking, W. Israel, Cambridge University Press, Cambridge, 1979, 581-638.

[41] Penrose R., Twistor geometry of conformal infinity, in The Conformal Structure of Space-Time, Lecture Notes in Phys., Vol. 604, Editors J. Frauendiener, H. Friedrich, Springer, Berlin, 2002, 113-121.

[42] Penrose R., Cycles of time. An extraordinary new view of the universe, Alfred A. Knopf, Inc., New York, 2010.

[43] Series C., The modular surface and continued fractions, J. London Math. Soc. 31 (1985), 69-80.

[44] Shiraishi M., Mota D.F., Ricciardone A., Arroja F., CMB statistical anisotropy from noncommutative gravitational waves, arXiv:1401.7936.

[45] Takasaki K., Painlevé-Calogero correspondence revisited, J. Math. Phys. 42 (2001), 1443-1473, math.QA/0004118.

[46] Tod P., Penrose's circles in the CMB and a test of inflation, Gen. Relativity Gravitation 44 (2012), 29332938, arXiv:1107.1421.

[47] van Suijlekom W.D., The noncommutative Lorentzian cylinder as an isospectral deformation, J. Math. Phys. 45 (2004), 537-556, math-ph/0310009.

[48] Wehus I.K., Eriksen H.K., A search for concentric circles in the 7-year WMAP temperature sky maps, Astrophys. J. Lett. 733 (2011), L29, 6 pages, arXiv:1012.1268.

[49] Yamazaki H., Hara T., Dirac decomposition of Wheeler-DeWitt equation in the Bianchi Class A models, Progr. Theoret. Phys. 106 (2001), 323-337, gr-qc/0101066.

[50] Zaldarriaga M., BICEP2 results: a view from the outside, Lecture given at the "Workshop on Primordial Gravitational Waves and Cosmology" (Burke Institute, Caltech, May 2014). 